Herz 2015 • 40:351-352

DOI 10.1007/s00059-015-4227-1

Online publiziert: 10. April 2015

(c) Urban \& Vogel 2015

\author{
B. Maisch \\ Emer. Direktor der Klinik für Innere Medizin - Kardiologie, UKGM GmbH \\ und Philipps-Universität Marburg
}

\title{
Sport und Herz - keine ganz problemlose Beziehung
}

„No sports“ - das soll Winston Churchill's legendäre Antwort gewesen sein, die er einem Reporter auf die Frage gegeben habe, wie er als passionierter Zigarrenraucher und Whiskeytrinker sein hohes Alter erreicht habe. Der am 30. November 1874 in Woodstock geborene englische Premierminister, der England durch den 2. Weltkrieg führte, verstarb gut 90 Jahre später am 24. Januar 1965 in London. Die Authentizität dieser oft zitierten Antwort ist ebenso wenig belegt, wie „seine“ Antwort aus medizinischer Sicht richtig sein kann.

„Fußball ist alles“ ist auf einem Plakat für den Bundesligaverein VfL Wolfsburg zu lesen. Sport als zentraler Lebensinhalt und nicht nur als „wichtigste Nebensache der Welt" wirft ein Schlaglicht nicht nur auf viele Profi- oder Amateursportler, sondern auch auf Fans und Zuschauer.

In dieser Gemengelage stellt sich das Thema dieses Heftes "Sport und Herz" als eine nicht ganz problemlose Beziehung dar, mit der sich Herz/Cardiovascular Diseases auch nicht zum ersten Mal beschäftigt. Bereits im Jahr 2009 befasste sich ein eigenes Heft von Herz mit Sport und plötzlichem Herztod. Im Jahr 2012 war "Sportkardiologie“ ein zentrales Thema. Und jetzt, drei Jahre später, steht das Thema erneut im Mittelpunkt eines Übersichtshefts. Zwar hat sich zwischen 2009 und 2015 viel im Sport und in der Kardiologie ereignet, doch einige Themen sind auch nach sechs Jahren noch immer aktuell, neue sind dazu gekommen.

Aktuell geblieben und weiterentwickelt hat sich die Bedeutung der Epigenetik. Darunter sind subsummiert Modifika- tionen am Chromatin, z. B. bei den posttranslationalen Histonmodifikationen und DNA-Methylierungen. Die Expression von mikro-RNA-Molekülen in der Pathogenese zahlreicher kardiovaskulärer Erkrankungen hat unserem Verständnis von Krankheit und Gesundheit eine weitere Dimension hinzugefügt. Philipp Zimmer und Wilhelm Bloch zeigen in ihrem Beitrag zu epigenetischen Anpassungen des Herz-Kreislauf-Systems, dass körperliche Aktivität als epigenetischer Regulator fungiert, der pathophysiologischen Entwicklungen im Herz-Kreislauf-System entgegenwirken, sie aber auch bei exzessiver Beanspruchung mitverursachen kann.

Simon Wernhart, Milan Dinic, Axel Pressler und Martin Halle weisen in ihrem Beitrag"Prävention kardiovaskulärer Erkrankungen durch Sport und körperliche Aktivität - eine Frage der Intensität?" darauf hin, dass körperliche Inaktivität und eingeschränkte körperliche Leistungsfähigkeit mit einer um bis zu $9 \%$ verfrühten Mortalität einhergehen können, während regelmäßige körperliche Aktivität sich positiv auf die kardiovaskuläre Morbidität und Mortalität auswirken dürfte. Während moderate körperliche Aktivität das kardiovaskuläre Risikoprofil durch Blutdrucksenkung, Erhöhung der Insulinsensitivität, Verbesserung des Lipidstoffwechsels, Reduktion chronischinflammatorischer Konstellationen und Gewichtsreduktion günstig beeinflusst, können bei höchstgradigen Belastungen wie beim Marathontraining auch negative Effekte induziert werden.

Beim „Sportherz“war das Augenmerk bislang meist auf akute und chronische
Veränderungen des systemischen Kreislauf und des linken Herzens gerichtet. Erst in den letzten Jahren wurden Veränderungen des rechten Herzens und die Interaktion des rechten mit dem linken Herzen näher untersucht. Antonello D’Andrea, André La Gerche, Enrica Golia, Roberto Padalino, Raffaele Calabrò, Maria Giovanna Russo und Eduardo Bossone zeigen in ihrem Beitrag, dass es unter pathophysiologischen Bedingungen zu rechtskardialen Veränderungen kommen kann, die zu atrialen und ventrikulären Arrhythmien prädisponieren. So kann es zum Phänotyp einer durch Leistungssport hervorgerufenen rechsventrikulären Kardiomyopathie kommen.

Bei Leistungssportlern wird in der öffentlichen Wahrnehmung und veröffentlichten Meinung meist kein erhöhtes Gesundheitsrisiko vermutet, es sei denn durch Sportverletzungen. Es kommt bei ihnen aber signifikant häufiger zu Fällen des plötzlichen Herztodes (PHT), worauf Tobias Tönnis, C. Tack und KarlHeinz Kuck in ihrem Beitrag zum PHT bei Sportlern hinweisen. Die Inzidenz des PHT bei Sportlern bewegt sich zwischen 0,6 und 3,0/100.000 pro Jahr und stellt keine vernachlässigbare Größe dar. Der PHT ist häufig die erste und leider auch letzte Manifestation einer zugrunde liegenden kardialen Grunderkrankung, die durch Screening erkannt werden könnte.

Deshalb sollte sich jeder Sportler vor Aufnahme eines Trainingsprogramms einem allgemeinen und zusätzlich für die gewählte Sportart speziellen diagnostischen Screening unterziehen, so Robert Skalik in seinem Beitrag über das Scree- 
ning von Athleten vor Beginn von Wettkampfsport oder Training. Welche medizinische Diagnostik routinemäßig vor einer Trainingsaufnahme durchgeführt werden soll, um bei außerordentlichen Belastungen die Gesundheit nicht zu gefährden und einem PHT vorzubeugen, wird in Europa und den USA kontrovers diskutiert. Diese medizinische Eintrittsuntersuchung mit körperlicher Untersuchung und Erhebung der Familienanamnese sowie mindestens ein Ruhe-EKG sind in Europa elementare Bestandteile einer Empfehlung zum Screening. In den USA wird dagegen auf das EKG verzichtet. Aber selbst ein Screening mit einem EKG in Ruhe garantiert nicht immer eine ausreichende diagnostische Sicherheit für Wettkampf- und Freizeitsportler. Und ob eine Empfehlung dann auch leitlinienkonform überhaupt durchgeführt wird, steht noch auf einem anderen Blatt.

\section{》) Jeder Sportler sollte sich vor Aufnahme eines Trainingsprogramms einem allgemeinen und zusätzlich für die gewählte Sportart speziellen diagnostischen Screening unterziehen}

Körperliches Training und Sport sind bewährte Bestandteile der Primär- und der Sekundärprävention bei koronarer Herzkrankheit sowie stabiler chronischer Herzinsuffizienz. Dagegen sind Wettkampfsport bei den meisten Formen einer hypertrophischen Kardiomyopathie (HCM) sowie Sport überhaupt bei Myokarditis und Perikarditis und bei rechtsventrikulärer Kardiomyopathie kontraindiziert. Auch Freizeitsportler sollten zu ihrer eigenen Sicherheit diese Erkrankungen ausschließen, bevor sie beginnen, Sport zu treiben. Auch Menschen, die sich zu einem körperlichen Training nach Jahren körperlicher Inaktivität im mittleren Lebensalter entschließen, sollten vorher einen Sportarzt oder Kardiologen konsultieren, bevor sie ihr Fitnesstraining aufnehmen, so Bernhard Maisch in seinem Beitrag über „Sport bei Herzpatienten und Athleten mit kardiovaskulärem Risiko - die Balance zwischen Nutzen und Ri- siko“. Die Forderung nach medizinischer Begleitung auch von Freizeitsportlern gilt besonders für Ausdauersportarten mit sehr hohem Trainings- und Leistungsanspruch wie Marathonlauf und Marathontraining sowie wettkampfmäßigem Radund Rudersport. Bei diesen extremeren Sportarten kann es auch bei ursprünglich gesunden Teilnehmern zu strukturellen Veränderungen im Herzmuskel kommen, die das Ziel der körperlichen Fitness vital gefährden. Außerdem wissen wir bis heute fast nichts über den Nutzen und das Risiko sportlicher Aktivität von Sportlern mit physischen Handicaps, die an Wettkampfsportarten bei paraolympischen Spielen teilnehmen, oder von Athleten mit geistiger Behinderung bei Wettkämpfen wie Special Olympics und Unified Sports. Diese Sportler finden auch in den Leitlinien bisher keine ausreichende Berücksichtigung.

Bei der rechtsventrikulären arrhythmogenen Kardiomyopathie (ARVC) beeinflussen Sport und körperliche Aktivität die Prognose ungünstig und können lebensbedrohlich sein. Aus diesem Grund empfehlen die Leitlinien, dass Patienten mit ARVC stärkere körperliche Belastung unabhängig vom Genotyp meiden sollen. Abhishek C. Sawant und Hugh Calkins erregten in der Fachpresse starke Aufmerksamkeit mit der Beobachtung, dass es eine erworbene Form der ARVC gibt, die allein durch körperliche Belastung und Sport verursacht wird und der deshalb als belastungsabhängiger ARVC Krankheitswert zukommen dürfte.

Doping ist die dunkle Seite des Sports, zu der Detlef Thieme und Andreas Büttner ihren analytischen und rechtsmedizinischen Kommentar geben. In ihrem Beitrag zu Schäden durch Doping zeigen sie eindrucksvoll, dass insbesondere der Missbrauch von anabol-androgenen Steroiden im professionellen sowie im Breiten- und Freizeitsport weit verbreitet ist und $\mathrm{zu}$ gravierenden Nebenwirkungen führt. Neben endokrinen Nebenwirkungen sind vor allem kardiovaskuläre Veränderungen eine nicht zu unterschätzende Folge des Dopings, wie durch forensische Untersuchungen von Todesfällen oder im Zusammenhang mit Straftaten zwar anekdotisch, aber dort anschaulich und überzeugend belegt werden kann.
Diese Ausgabe von Herz zu den physiologischen und pathophysiologischen Themen von Sport und körperlicher Aktivität zeigt die Breite der Diskussion und den Spannungsbogen der aktuellen Problemfelder auf. Auch für den Sport von Gesunden und Kranken gilt das Prinzip des Arztes und Alchemisten Paracelsus (1493-1541), der eigentlich Philippus Aureolus Theophrastus Bombastus von Hohenheim hieß. Es ist uns seit Jahrhunderten vertraut: „Dosis facit venenum“.

Bernhard Maisch

\section{Korrespondenzadresse}

Prof. Dr. B. Maisch

Emer. Direktor der Klinik für Innere Medizin Kardiologie,

UKGM GmbH und Philipps-Universität Marburg Feldbergstr. 45, 35043 Marburg

bermaisch@gmail.com

Interessenkonflikt. B. Maisch gibt an, dass kein Interessenkonflikt besteht. 